\title{
Mielinólise Extrapontina em Adolescente com Diabetes Insípidus Secundário a Disgerminoma do Sistema Nervoso Central: Relato de Caso
}

doi: https://doi.org/10.32635/2176-9745.RBC.2018v64n3.55

Extra-Pontine Myelinolysis in a Patient with Diabetes Insipidus Secondary to Disgerminoma of the Central Nervous

System: Case Report

Mielinólise Extrapontina en Paciente con Diabetes Insípida Secundário a Disgerminoma del Sistema Nervioso

Central: Relato de Caso

Bruno Espírito Santo de Araújo'; Daniela Capuzzo Dias Castiglione²; Fernanda Lobo Rascão³; Sandra Helena dos Santos Victal ${ }^{4}$; Sima Esther Ferman ${ }^{5}$; Fernanda Ferreira da Silva Lima ${ }^{6}$; Fernanda Costa Capela ${ }^{7}$

\section{Resumo}

Introdução: A síndrome de desmielinização osmótica é uma condição neurológica rara causada pelo dano à bainha de mielina dos neurônios, com difícil manejo do distúrbio do sódio em paciente com diabetes insípidus. Relato do caso: Adolescente do sexo feminino, 14 anos, com diabetes insípidus secundária a disgerminoma do sistema nervoso central, com hiponatremia grave (sódio $103 \mathrm{mEq} / \mathrm{L}$ ). Cinco dias após a correçáo rápida do sódio, apresentou coma (Escala de Glasgow:11), disfagia, mutismo e tetraparesia. Os achados na ressonância nuclear magnética craniana foram compatíveis com diagnóstico de mielinólise extrapontina. Vinte e cinco dias após a internação no Centro de Tratamento Intensivo, a paciente encontrava-se lúcida, orientada, deambulando sem dificuldade, alimentando-se por via oral, sem engasgos, ainda com discreta diminuição de força nos membros superiores e comunicação verbal pouco lentificada. A ressonância nuclear magnética após três meses mostrou atrofia dos núcleos da base, comprovando lesão celular grave. Conclusáo: A desmielinização osmótica pode apresentar-se com formas clínicas leves ou assintomáticas, até sequelas motoras graves e morte. Não existe tratamento específico, o que ressalta a importância do diagnóstico precoce e do manejo adequado do distúrbio do sódio, assim como controle rigoroso dos seus níveis séricos.

Palavras-chave: Mielinólise Central da Ponte; Sistema Nervoso Central; Diabetes Insípido; Hiponatremia.

\begin{abstract}
Introduction: Osmotic demyelination syndrome is a rare neurological condition caused by damage to the myelin sheath of neurons, involving difficulty in the management of sodium imbalance in patients with diabetes insipidus. Case report: Patient was a 14-year-old female with diabetes insipidus secondary to dysgerminoma of the central nervous system, with severe hyponatremia (sodium $103 \mathrm{mEq} / \mathrm{L}$ ). Five days after rapid correction of the sodium imbalance, the patient presented coma (Glasgow scale:11), dysphagia, mutism, and quadriparesis. Cranial MRI findings were consistent with a diagnosis of extrapontine myelinolysis. Twenty-five days after admission to the ICU, the patient was alert, oriented, walking without difficulty, eating an oral diet without choking, although with slightly diminished strength in the upper limbs and slightly sluggish verbal communication. Three-month follow-up MRI showed atrophy of the basal nuclei, confirming severe cellular injury. Conclusion: Presentation of osmotic demyelination may range from mild or asymptomatic clinical forms to severe motor sequelae and death. There is no specific treatment, which highlights the importance of early diagnosis and adequate management of the sodium imbalance, as well as rigorous control of serum sodium levels. Key words: Myelinolysis, Central Pontine; Central Nervous System; Diabetes Insipidus; Hyponatremia.
\end{abstract}

Resumen

Introducción: La síndrome de desmielinización osmótica es una condición neurológica rara causada por el daño en lás vainas de mielina de las neuronas, con difícil manejo del trastorno de los niveles de sódio en los pacientes con diabetes insípida. Relato del caso: Adolescente do sexo feminino, 14 anos, com diabetes insípida secundária a disgerminoma del sistema nervioso central, con hiponatriemia grave (sódio $103 \mathrm{mEq} / \mathrm{L}$ ). Cinco días después de la corrección rápida del sódio, presentó coma (Escala de Glasgow: 11), disfagia, mutismo y tetraparesia. Los hallazgos en la resonancia magnética del cráneo fueron compatibles con diagnosis de mielinólise extra-pontina. Veinticinco días después de la internaciónen el Centro de Tratamiento Intensivo la paciente se encontraba lúcida, orientada, deambulando sin dificultad, alimentándose por vía oral sin atorarse, aúncon discreta disminución de fuerza en los miembros superiores y comunicación verbal poco lentificada. Una resonancia nuclear magnética después de tres meses mostró atrofia de los núcleos de la base del cráneo, comprobando lesión celular grave. Conclusión: Una síndrome de desmielinización osmótica puede presentarse con formas clínicas ligeras o asintomáticas, hasta secuelas motoras graves y muerte. No existe tratamiento específico, lo que resaltala importancia del diagnóstico precoz y del manejo adecuado del disturbio del sodio, así como control riguroso de sus niveles séricos. Palabras clave: Mielinólisis Pontino Central; Sistema Nervioso Central; Diabetes Insípida; Hiponatremia.

\footnotetext{
1 Instituto Nacional de Câncer José Alencar Gomes da Silva (INCA). Rio de Janeiro (RJ), Brasil. Orcid iD: https://orcid.org/0000-0002-7480-1973

${ }^{2}$ Universidade do Estado do Rio de Janeiro. Rio de Janeiro (RJ), Brasil. Orcid iD: https://orcid.org/0000-0002-3055-0148

${ }^{3}$ INCA. Rio de Janeiro (RJ), Brasil. Orcid iD: https://orcid.org/0000-0002-1456-3205

${ }^{4}$ INCA. Rio de Janeiro (RJ), Brasil. Orcid iD: https://orcid.org/0000-0001-6683-1059

${ }^{5}$ INCA. Rio de Janeiro (RJ), Brasil. Orcid iD: https://orcid.org/0000-0002-7076-6779

${ }^{6}$ INCA. Rio de Janeiro (RJ), Brasil. Orcid iD: https://orcid.org/0000-0002-6658-3101

${ }^{7}$ INCA. Rio de Janeiro (RJ), Brasil. Orcid iD: https://orcid.org/0000-0003-3979-9801

Endereço para correspondência: Bruno Espírito Santo de Araújo. Hospital do Câncer I. INCA. Praça Cruz Vermelha, 23 - 5ªndar (chefia da pediatria) - Centro. Rio de Janeiro (RJ), Brasil. CEP 20230-130. E-mail: brunoespirito@hotmail.com.
} 


\section{INTRODUÇÃO}

A síndrome de desmielinização osmótica é uma condição neurológica rara causada por dano à bainha de mielina dos neurônios ${ }^{1}$, relacionada à mudança súbita na osmolaridade plasmática, em especial do sódio sérico. A apresentação clássica é de mielinólise central pontina, com acometimento da substância branca dessa regiáo, e a mielinólise extrapontina que pode acometer amplas áreas do encéfalo como os pedúnculos cerebelares, núcleos da base, substância branca frontal e temporal, cápsula externa e interna, tálamo, núcleo subtalâmico, camadas profundas do córtex cerebral, hipocampo e corpo caloso ${ }^{2}$. Ocorre esporadicamente em todas as idades, em ambos os sexos e sua incidência exata é desconhecida ${ }^{3}$.

O relato de caso tem por objetivo descrever um caso de desmielinização osmótica, bem como destacar a dificuldade de manejo no distúrbio do sódio em paciente com diabetes insípidus, assim como a importância de um controle rigoroso da natremia nesses pacientes.

O presente estudo foi aprovado pelo Comitê de Ética em Pesquisa do Instituto Nacional de Câncer José Alencar Gomes da Silva (CEP-INCA), sob o número CAAE: 54322015.7.0000.5274. A autorização para publicação foi fornecida pelos responsáveis, por meio de assinatura do Termo de Consentimento Livre e Esclarecido.

\section{RELATO DO CASO}

Paciente do sexo feminino, 14 anos, com diagnóstico de disgerminona do sistema nervoso central, suprasselar, pan-hipopituitarismo, em uso regular de levotiroxina, prednisona e desmopressina (DDAVP). Em tratamento quimioterápico há sete meses, com último ciclo de quimioterapia há 13 dias. Procurou atendimento médico por febre, vômitos e baixa ingesta hídrica, foi diagnosticada neutropenia febril e hipernatremia (sódio sérico de 160 $\mathrm{mEq} / \mathrm{L}$ ). Admitida na enfermaria para tratamento com antimicrobiano e correção do distúrbio do sódio com reposição de água livre. O sódio foi corrigido e completado o esquema antibiótico.

No décimo primeiro dia de internação, apresentou alteração do nível de consciência, com diagnóstico de hiponatremia grave (sódio $103 \mathrm{mEq} / \mathrm{L}$ ), sendo transferida para Centro de Tratamento Intensivo (CTI) pediátrico, onde iniciou correçấo do distúrbio hidroeletrolítico com solução salina a $3 \%$.

No exame físico de admissão no CTI pediátrico, a paciente encontrava-se sonolenta, respondendo às solicitaçóes, mobilizando os quatro membros, pupilas isocóricas e fotorreagentes, estável hemodinamicamente, com ausculta respiratória sem ruídos adventícios. Iniciou correção da hiponatremia com solução salina a 3\%, calculada para corrigir $10 \mathrm{mEq}$ em 24 horas, e avaliada por coleta seriada de sódio sérico a cada três horas.

Algumas horas após início da correção, a paciente melhorou o nível de consciência, mantendo-se vígil, com movimentação dos quatro membros e resposta verbal adequada. Porém, mesmo com o controle do nível sérico a cada três horas, o sódio aumentou $18 \mathrm{mEq} / \mathrm{L}$ (sódio $121 \mathrm{mEq} / \mathrm{L}$ ) em 12 horas. Nesse momento, foi suspensa a reposição de sódio e administrado DDAVP (intranasal). Nas horas subsequentes, fez-se reposição de água livre e administração do DDAVP. A despeito disso, o sódio continuou subindo, chegando a $138 \mathrm{mEq} / \mathrm{L}$, com aumento de $35 \mathrm{mEq} / \mathrm{L}$ em 24 horas e $54 \mathrm{mEq} / \mathrm{L}$ em 48 horas (Figura 1).

Sódio sérico

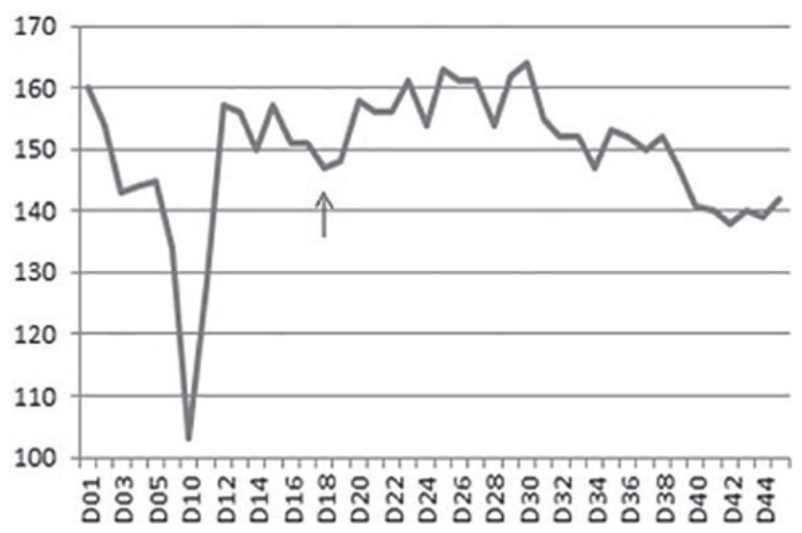

Figura 1. Nível sérico de sódio nos primeiros dias de internação no CTI pediátrico, seta destaca momento de piora clínica

Cinco dias após a hiponatremia (sódio $103 \mathrm{mEq} / \mathrm{L}$ ), a paciente apresentou piora neurológica, com rebaixamento do nível de consciência, Glasgow 11 (abertura ocular aos chamados, palavras incompreensíveis e obedecia a comandos), disfagia, parestesia nos quatro membros, hipoventilaçáo com queda de língua e necessitou de suporte ventilatório não invasivo (nesse dia, sódio 149 $\mathrm{mEq} / \mathrm{L}$ ). Dois dias após, foi feita ressonância nuclear magnética (RNM), que evidenciou realce acometendo o núcleo lentiforme bilateral e difusamente a substância branca subcortical, sugestiva de mielinólise extrapontina grave (Figura 2).

Nos dias subsequentes, apresentou oscilação do nível de consciência, tetraparesia, tentativa de comunicação com examinador, emitindo sons incompreensíveis, comunicando-se por meio de movimentação dos olhos, sugestivo de síndrome de encarceramento.

Vinte e cinco dias após internação no CTI pediátrico, iniciou melhora neurológica progressiva, com mobilização 


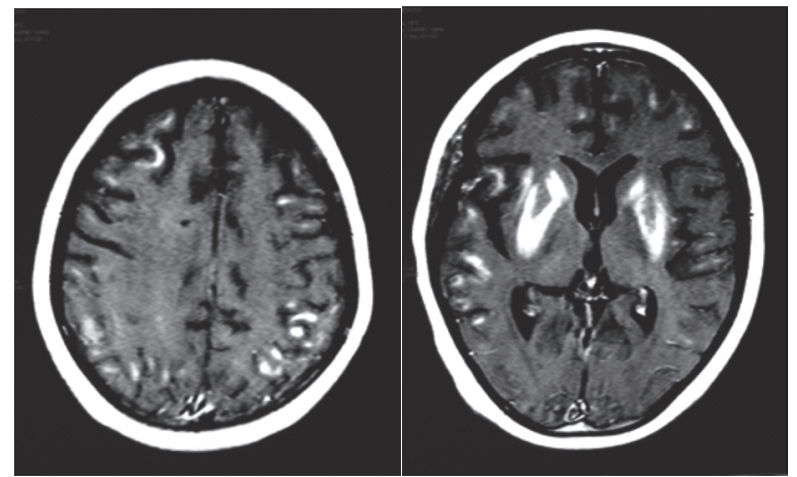

Figura 2. RNM plano axial, em T1 com contraste. Realce acometendo o núcleo lentiforme bilateral e difusamente a substância branca subcortical

dos quatro membros, porém com fraqueza muscular, respondendo a solicitaçôes simples com palavras isoladas, com coerência e melhora da disfagia, e início da dieta por via oral. Foi transferida para enfermaria no trigésimo quarto dia de acompanhamento em nosso setor.

Três meses após o início do quadro, foi realizada nova RNM (Figura 3) que não evidenciou realce, com atrofia dos núcleos da base, formação de cavitação e alargamento da cissura de Sylvios bilateralmente. No exame clínico, encontrava-se lúcida, orientada, deambulando sem dificuldade, alimentando-se por via oral sem engasgos, com discreta diminuição de força nos membros superiores e comunicação verbal ainda lentificada.

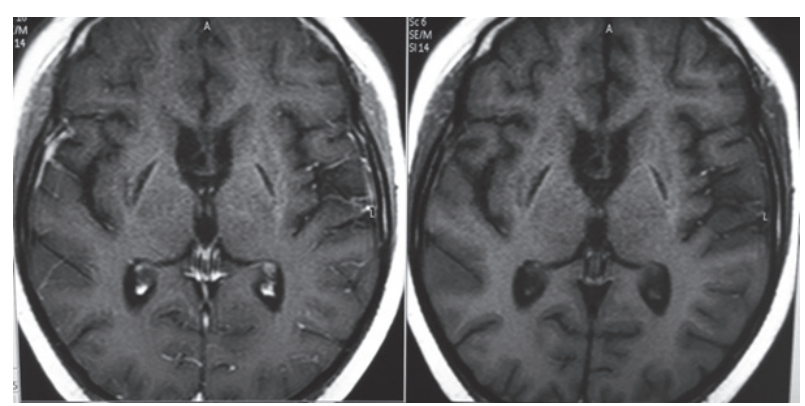

Figura 2. RNM crânio, corte axial, Tl com e sem contraste respectivamente

\section{DISCUSSÃO}

A síndrome de desmielinização osmótica está tradicionalmente associada com mudanças rápidas da osmolaridade sérica, em particular à correção rápida da hiponatremia. No entanto, várias outras associaçóes têm sido relatadas, tais como hipofosfatemia grave, hipocalemia, diabetes mellitus, insuficiência renal, hemodiálise, hiperemêse gravídica, anorexia nervosa, doença de Wilson, queimaduras graves e lúpus eritematoso sistêmico, entre outras ${ }^{1}$.
A hiponatremia é definida como um sódio sérico menor que $135 \mathrm{mEq} / \mathrm{L}$ e é considerada severa quando menor que $120-125 \mathrm{mEq} / \mathrm{L}^{3,4}$. A desmielinização osmótica está relacionada com uma correção rápida da hiponatremia superior a 10-12 mEq/L em 24 horas; ou seja, maior que a velocidade de 0,5 a $1 \mathrm{mEq} / \mathrm{L} / \mathrm{h}$ ou $25 \mathrm{mEq} / \mathrm{L}$ em 48 horas $^{4}$.

No caso descrito, a paciente apresentou rápida correção da hiponatremia, com aumento de $34 \mathrm{mEq} / \mathrm{l}$ em 24 horas e $54 \mathrm{mEq} / \mathrm{l} \mathrm{em} 48$ horas, evoluindo com hipernatremia a despeito do controle rigoroso do sódio sérico e das inúmeras tentativas de reposição de água livre e uso de DDAVP, o que destaca a dificuldade no manejo em razáo do diabetes insipidus associado e sua estreita relação com disgerminoma intracraniano, mesmo após o tratamento cirúrgico e quimioterápico 5 .

Classicamente, a desmielinização osmótica apresenta-se com um curso bifásico, fase inicial com convulsão ou encefalopatia, com melhora progressiva dos sintomas neurológicos com a correção da hiponatremia, e uma fase mais tardia, ocorrendo após dois a oito dias, com deterioração grave expressa por flutuação do nível de consciência, mudanças de comportamento, disartria, mutismo, disfagia, disfunção oculomotora e graus variáveis de tetraparesia. A tetraparesia inicialmente é flácida por dano do trato corticoespinhal e progride para fase espástica com lesão da base da ponte. Os casos mais graves podem apresentar síndrome do encarceramento, com os pacientes acordados, mas incapazes de se mover ou se comunicar, apenas com a preservação dos movimentos oculares verticais e piscar dos olhos ${ }^{1,6}$.

A paciente do caso apresentou quadro clínico clássico, com rebaixamento do nível de consciência, disfagia, disartria e diminuição de força no quinto dia após distúrbio do sódio. O diagnóstico de síndrome de desmileinizaçáo osmótica foi confirmado com a RNM, com lesôes extrapontinas graves. Nos dias subsequentes, ainda manifestou piora clínica progressiva com mutismo e tetraparesia. Observou-se início da melhora clínica no vigésimo quinto dia de internação no CTI pediátrico.

A RNM do encéfalo é sensível para a detecção de mielinólise com reconhecimento dos casos leves e até assintomáticos, além de determinar a extensão da desmielinização. Os achados radiológicos típicos são lesôes simétricas e hipointensas nas imagens em T1, na ponte, poupando a periferia; e outras estruturas extrapontinas, e lesôes hiperintensas nas imagens em T2 e Fluid Acquisition Inversion Recovery (Flair) $^{6}$. No caso descrito, a RNM evidenciou realce nos núcleos da base e difusamente na substância branca subcortical em T1, T2 e Flair, que caracteriza lesão grave com morte celular.

Alguns trabalhos relatam que os achados radiológicos típicos de mielinólise pontina não costumam ser vistos 
na RNM na primeira semana após o início dos sintomas clínicos ${ }^{7}$. Atualmente, a modalidade de imagem ponderada em difusão é a mais utilizada, em razão da detecção precoce dos achados de mielinólise, ocorrendo 24 horas após início da tetraplegia ${ }^{2,6}$. Porém, nesse caso, a RNM foi realizada no sétimo dia e não evidenciou restrição na sequência ponderada em difusão, o que pode significar que, além da desmielinização, houve morte celular.

Nas fases subaguda e crônica, as lesôes pontinas e, em particular, extrapontinas podem se tornar menores e mais bem definidas. Em alguns casos, a RNM pode revelar a lesão típica em asa de morcego na base da ponte ${ }^{2}$. A RNM de controle mostrou atrofia dos núcleos da base, comprovando lesão celular grave.

A literatura médica descreve uma evolução variável de desmielinização osmótica que vai desde a recuperação total até o óbito. Em geral, a recuperação é lenta e gradativa. A mortalidade associada à hiponatremia grave situa-se entre $40 \%$ e $50 \%{ }^{8,9}$. Em 2006, foi descrito em nosso meio um caso de mielinólise extrapontina em adolescente com desfecho desfavorável ${ }^{2}$. Uma revisão da literatura, realizada em 2014, observou que mais da metade dos casos de síndrome da desmielinização osmótica podem apresentar boa recuperação, mesmo em pacientes com déficits neurológicos graves iniciais. Pacientes pós-transplante hepático apresentaram pior prognóstico ${ }^{1}$.

Apesar da gravidade do acometimento neurológico inicial, a paciente apresentou boa evolução clínica, com recuperação parcial da força, melhora da disfagia e disartria.

\section{CONCLUSÃO}

A síndrome de desmielinização osmótica é uma condição neurológica rara, com clínica de acometimento leve ou assintomático até sequelas motoras graves e morte. Não existe tratamento específico, o que ressalta a importância do diagnóstico precoce do distúrbio do sódio e manejo adequado, bem como o controle rigoroso e seriado de seus níveis séricos.

Estudos em pediatria são necessários para estabelecer protocolos mais seguros de controle e manejo para os doentes com sódio sérico em níveis críticos.

\section{CONTRIBUIÇÕES}

Bruno Espírito Santo de Araújo, Daniela Capuzzo Dias Castiglione e Sandra Helena dos Santos Victal participaram substancialmente da concepção e do planejamento do estudo; da análise e da interpretação dos dados da pesquisa; assim como da redação, revisão crítica e aprovação final da versão para publicação. Fernanda
Lobo Rascão, Sima Esther Ferman, Fernanda Ferreira da Silva Lima e Fernanda Costa Capela participaram substancialmente da análise e da interpretação dos dados da pesquisa; assim como da redação, revisão crítica e aprovação final da versão para publicação.

\section{DECLARAÇÃO DE CONFLITO DE INTERESSES}

Nada a declarar.

\section{FONTES DE FINANCIAMENTO}

Não há.

\section{REFERÊNCIAS}

1. Singh TD, Fugate JE, Rabinstein AA. Central pontine and extrapontine myelinolysis: a systematic review. Eur J Neurol. 2014;21(12):1443-1450.

2. Brito AR, Vasconcelos MM, Cruz LC Jr, Oliveira ME, Azevedo AR, Rocha LG, et al. Central pontine and extrapontine myelinolysis: report of a case with a tragic outcome. J Pediatr. 2006;82(2):157-160.

3. Ranger A, Szymczak A, Levin S, Salvadori M, Fraser DD. Osmotic myelinolysis with malignant cerebellar edema occurring after DDAVP-induced hyponatremia in a child. Pediatr Neurosurg. 2010;46(4):318-323.

4. Harring TR, Deal NS, Kuo DC. Disorders of sodium and water balance. Emerg Med Clin North Am. 2014;32(2):379-401.

5. Jorsal T, Rorth M. Intracranial germ cell tumours. A review with special reference to endocrine manifestations. Acta Oncol. 2012;51(1):3-9.

6. Alleman AM. Osmotic demyelination syndrome: central pontine myelinolysis and extrapontine myelinolysis. Semin ultrasound CT MR. 2014;35(2):153-159.

7. Neto PG, Neri VC. Síndrome de desmielinização osmótica em paciente jovem, com hiponatremia e mau prognóstico. Rev Cient Fac Med Campos. 2007;2(2):30-36.

8. Jurno ME, Castro MH, Lage MA, Dupin JH, Paula AJ, Bello GV. Osmotic demyelination syndrome: report of a case with favorable outcome. Radiol Bras. 2012;45(1):61-62.

9. Huq S, Wong M, Chan H, Crimmins D. Osmotic demyelination syndromes: central and extrapontine myelinolysis. J Clin Neurosci. 2007;14(7):684-688. 